\title{
PHOTOELECTRIC OBSERVATIONS OF SOUTHERN CEPHEIDS IN 2001
}

\author{
LEONID N. BERDNIKOV ${ }^{1}$ \\ Sternberg Astronomical Institute and Isaac Newton Institute of Chile, Moscow Branch, 13 Universitetskij prosp., \\ Moscow 119899, Russia; berdnik@sai.msu.ru \\ AND \\ DAVID G. TURNER \\ Department of Astronomy and Physics, Saint Mary’s University, Halifax, NS B3H 3C3, Canada; turner@ap.stmarys.ca \\ Received 2001 April 23 ; accepted 2001 August 3
}

\begin{abstract}
A total of 2097 photometric observations in the $B V I_{c}$ system are presented for 117 Cepheids located in the southern hemisphere. The main purpose of the photometry is to provide new epochs of maximum brightness for studying Cepheid period changes, as well as to establish current light elements for the Cepheids.

Subject headings: Cepheids - stars: fundamental parameters - surveys - techniques: photometric

On-line material: machine-readable table
\end{abstract}

\section{INTRODUCTION}

Regular photometric monitoring of Cepheid variables is important for a number of reasons. The complete phase coverage of Cepheid light curves observed over many cycles is essential for establishing reliable mean magnitudes and colors for individual objects, while seasonal checks on the phasing of their light variations allow one to detect regular changes in pulsation period (e.g., Berdnikov 1994; Berdnikov \& Pastukhova 1994a, 1994b, 1995; Berdnikov et al. 1997a, 1997b). Regular monitoring is also crucial for detecting irregular changes in Cepheid light curves as well as for correctly phasing the brightness changes with radial velocity variations in the same objects, an essential first step in Baade-Wesselink studies of Cepheids. Excellent compilations of data related to all such studies have been provided previously by Szabados $(1977,1980,1981,1989,1991)$ and others.

Since Cepheids are evolving through the instability strip in the H-R diagram, one expects their pulsation periods to change in regular fashion as a consequence of their changing mean radii. Stars evolving toward the cool side of the $\mathrm{H}-\mathrm{R}$ diagram at nearly constant luminosity are slowly increasing in size and should exhibit increasing pulsation periods. Stars evolving toward the hot side of the H-R diagram at nearly constant luminosity are slowly decreasing in size and should exhibit decreasing pulsation periods. Both expectations are confirmed by the observed rates of period change in Cepheid pulsation periods (e.g., Berdnikov \& Ignatova 2000; Turner 1998; Turner et al. 1999; Turner et al. 2001), which prove to be very closely matched to the results expected from stellar evolutionary models. Superposed on such regular long-term changes are other effects, which include light travel time differences resulting from orbital motion about companions (see, e.g., Szabados 1977, $1980,1981,1989)$ as well as random fluctuations in period (Berdnikov et al. 2000; Turner et al. 2001). The latter phenomenon is well documented in cooler classes of pulsating stars (Eddington \& Plakidis 1929; Percy et al. 1997; Percy \& Hale 1998; Percy \& Colivas 1999) but is relatively unstudied in Cepheids themselves (see Turner et al. 2001). \footnotetext{
tories.

${ }^{1}$ Visiting Astronomer, Mount Stromlo and Siding Spring Observa-
}

A proper study of Cepheid period variations requires a sufficiently long baseline of observations with reasonably thorough temporal coverage in order to sort out clearly the various factors that affect period variations in the stars. Evolutionary effects generally produce a slow but regular increase or decrease in period that is most easily detected in those stars where evolution is occurring rapidly (such as at long pulsation periods or for first crossings of the instability strip). Light travel time effects are normally difficult to establish within the standard constraints associated with observational scatter but can be detected in data sets of sufficiently good quality. Random fluctuations in period, on the other hand, can produce very obvious effects on Cepheid $O-C$ diagrams (Turner et al. 1999; Berdnikov et al. 2000), which plot the differences between Observed and Computed times of light maximum from a linear ephemeris. To parameterize such effects properly requires the monitoring of Cepheid light variations on a fairly regular basis.

Up until a few years ago, Milky Way Cepheids were objects of moderately regular photometric observation. There was some bias toward the northern hemisphere sample at the expense of southern hemisphere objects, engendered by the concentration of photometric observing facilities in the northern hemisphere. That situation has since changed, primarily as a consequence of shifts in observing priorities at world observatories. For the last 5 or 6 years the main contribution toward regular monitoring of Cepheid variables in the Milky Way has come from programs of observation by the present authors (Berdnikov \& Vozyakova 1995; Berdnikov \& Turner 1995a, 1995b, 1998a, 1998b, 2000a, 2000b). In previous papers we provided large databases of observations obtained from photoelectric monitoring of southern hemisphere Cepheids, for which the temporal baseline is less complete than for their northern hemisphere kin. In this paper we add to that database with photoelectric observations obtained during 2001 February and March.

\section{OBSERVATIONAL DATA}

All observations presented here were made at Siding Spring Observatory, Australia, between 2001 February 2 and March 4. The data were obtained using the observatory's 24 inch reflector in combination with a photometer 
TABLE 1

New Photoelectric Observations of Southern CePheids.

\begin{tabular}{|c|c|c|c|c|c|c|c|c|c|}
\hline HJD 2,451,000+ & Phase & $V$ & $B-V$ & $V-I_{c}$ & HJD 2,451,000+ & Phase & $V$ & $B-V$ & $V-I_{c}$ \\
\hline \multicolumn{10}{|c|}{ T Ant } \\
\hline 948.0571 & 0.445 & 9.438 & 0.804 & 0.874 & 962.0806 & 0.822 & 9.664 & 0.843 & 0.925 \\
\hline 949.0510 & 0.613 & 9.633 & 0.915 & 0.960 & 963.0040 & 0.979 & 8.893 & 0.485 & 0.596 \\
\hline 949.2293 & 0.644 & 9.658 & 0.970 & 0.956 & 963.2137 & 0.015 & 8.894 & 0.482 & 0.620 \\
\hline 950.1386 & 0.798 & 9.714 & 0.921 & 0.948 & 964.0261 & 0.152 & 9.082 & 0.628 & 0.741 \\
\hline 951.9936 & 0.112 & 9.017 & 0.576 & 0.693 & 964.1985 & 0.182 & 9.134 & 0.659 & 0.762 \\
\hline 953.0629 & 0.294 & 9.276 & 0.765 & 0.839 & 964.9780 & 0.314 & 9.335 & 0.755 & 0.846 \\
\hline 953.0825 & 0.297 & 9.277 & 0.752 & 0.844 & 968.9252 & 0.983 & 8.885 & 0.494 & 0.611 \\
\hline 954.0268 & 0.457 & 9.441 & 0.818 & 0.882 & 969.1567 & 0.022 & 8.898 & 0.504 & 0.641 \\
\hline 957.0246 & 0.965 & 8.939 & 0.513 & 0.632 & 969.9466 & 0.156 & 9.095 & 0.627 & 0.722 \\
\hline 958.0022 & 0.131 & 9.041 & 0.607 & 0.709 & 971.9883 & 0.502 & 9.542 & 0.853 & 0.903 \\
\hline 958.1554 & 0.157 & 9.100 & 0.626 & 0.741 & 972.1070 & 0.522 & 9.529 & 0.862 & 0.925 \\
\hline 959.0301 & 0.305 & 9.299 & 0.750 & 0.842 & 972.9306 & 0.662 & 9.671 & 0.931 & 0.955 \\
\hline 959.1770 & 0.330 & 9.322 & 0.781 & 0.866 & 973.0692 & 0.685 & 9.728 & 0.909 & 0.967 \\
\hline
\end{tabular}

Note.-Table 1 is available in its entirety in the electronic edition of the Astrophysical Journal Supplement. A portion is shown here for guidance regarding its form and content.

designated as the "Motorized Filter Box" unit, a single channel instrument with a computer-controlled filter wheel. The photometer is a cooled unit containing a Hamamatsu GaAs photomultiplier tube that was used with $B V I_{c}$ filters chosen to match the Kron-Cousins photometric system (Cousins 1976). To allow for nonlinearity in the recording system, the following formula was used:

$$
N=N^{\prime}\left(1+d N^{\prime}\right),
$$

where $N^{\prime}$ and $N$ correspond to the recorded and true number of pulses received from the star per unit time interval, and $d$ is dead time of the instrument, which in our case was $25 \mathrm{~ns}$. Measurements of a number of Bright Star Catalogue stars up to magnitude 4 in $V$ indicate that equation (1) is reasonably good in this magnitude range.

At the beginning of each night we measured the atmospheric extinction using a selection of photometric standards from the $E$ regions (Menzies et al. 1989), namely stars numbered E130, E146, E229, E252, E388, E393, E429, E496, E577, E584, E633, E651, E739, and E746. The color extinction coefficients were taken to be $-0.0055,-0.02$, and 0.0 for $V$ magnitude, $B-V$ color, and $V-I_{c}$ color, respectively. In our calculations of air mass, the Forbes coefficient was taken to be -0.02 for the $B-V$ color and zero for the $V$ magnitude and $V-I_{c}$ color index (Straizys 1977).

Our measurements of the $\mathrm{E}$ region standards listed above were also used to determine the coefficients $\xi_{V}, \mu_{V}, \xi_{B-V}$, $\mu_{B-V}, \xi_{V-I_{c}}$, and $\mu_{V-I_{c}}$ for transformation of the instrumental quantities $v, b-v$, and $v-i_{c}$ to the standard KronCousins system:

$$
\begin{gathered}
V=v+\xi_{V}(B-V)+\mu_{V}, \\
B-V=\xi_{B-V}(b-v)+\mu_{B-V}, \\
\left(V-I_{c}\right)=\xi_{V-I_{c}}\left(v-i_{c}\right)+\mu_{V-I_{c}} .
\end{gathered}
$$

Mean transformation coefficients of $\xi_{V}=0.057 \pm 0.001$, $\xi_{B-V}=0.983 \pm 0.001$, and $\xi_{V-I_{c}}=1.068 \pm 0.001$ were determined from observations made on the best nights. They were used to define the zero points in $V, B-V$, and $V-I_{c}$ each one of 1.5 hours of every night from observations of the same standards using relations (2).

The photometric standards and bright Cepheids were observed through a $30^{\prime \prime}$ aperture, while the rest of the stars were observed through a $15^{\prime \prime}$ aperture. Since some of the stars are very bright, a metal film neutral density filter (Oriel 50550) was placed in front of the filter wheel to reduce the brightness of those stars.

\section{RESULTS AND DISCUSSION}

We obtained a total of 2097 observations for 117 Cepheids during the course of the observing run. The data are summarized in Table 1 and are plotted in Figures 1-16, where the phases were calculated using current elements for the Cepheids from Berdnikov \& Caldwell (2001). The exceptions are the four Cepheids listed in Table 2, for which the elements were newly calculated for the present paper.

The observational uncertainties in the data are close to $\pm 0.01 \mathrm{mag}$ in all bands. The large scatter in the data points for the light curves of Y Car (Fig. 2), GZ Car (Fig. 4), UZ Cen (Fig. 5), BK Cen (Fig. 6), VX Pup (Fig. 10), U TrA (Fig. 14), and AP Vel (Fig. 15) can be explained by the bimodality of those Cepheids, which exhibit pulsation in more than one mode simultaneously.

It is our intent to use the data tabulated here as part of more detailed future studies of the period changes in southern hemisphere Cepheids. Such data serve many purposes, however, so they are published here for the use of other researchers.

The authors gratefully acknowledge partial support for this work by research funding awarded through the Russian Foundation of Basic Research (RFBR) and the State Science and Technology Program "Astronomy" to L. N. B., and through the Natural Sciences and Engineering Research Council of Canada (NSERC) to D. G. T.

TABLE 2

Adopted ElEMENTS FOR Four CePHEIDS.

\begin{tabular}{cccc}
\hline \hline Cepheid & Initial Epoch & Period $(d)$ & Type \\
\hline EY Car ...... & 2451958.04 & 2.8763 & DCEP \\
HQ Car ...... & 2451970.06 & 14.02103 & CWA \\
ST Pup ...... & 2451968.23 & 18.551 & CWA \\
CO Pup ...... & 2451962.5 & 16.0324 & CWA \\
\hline
\end{tabular}



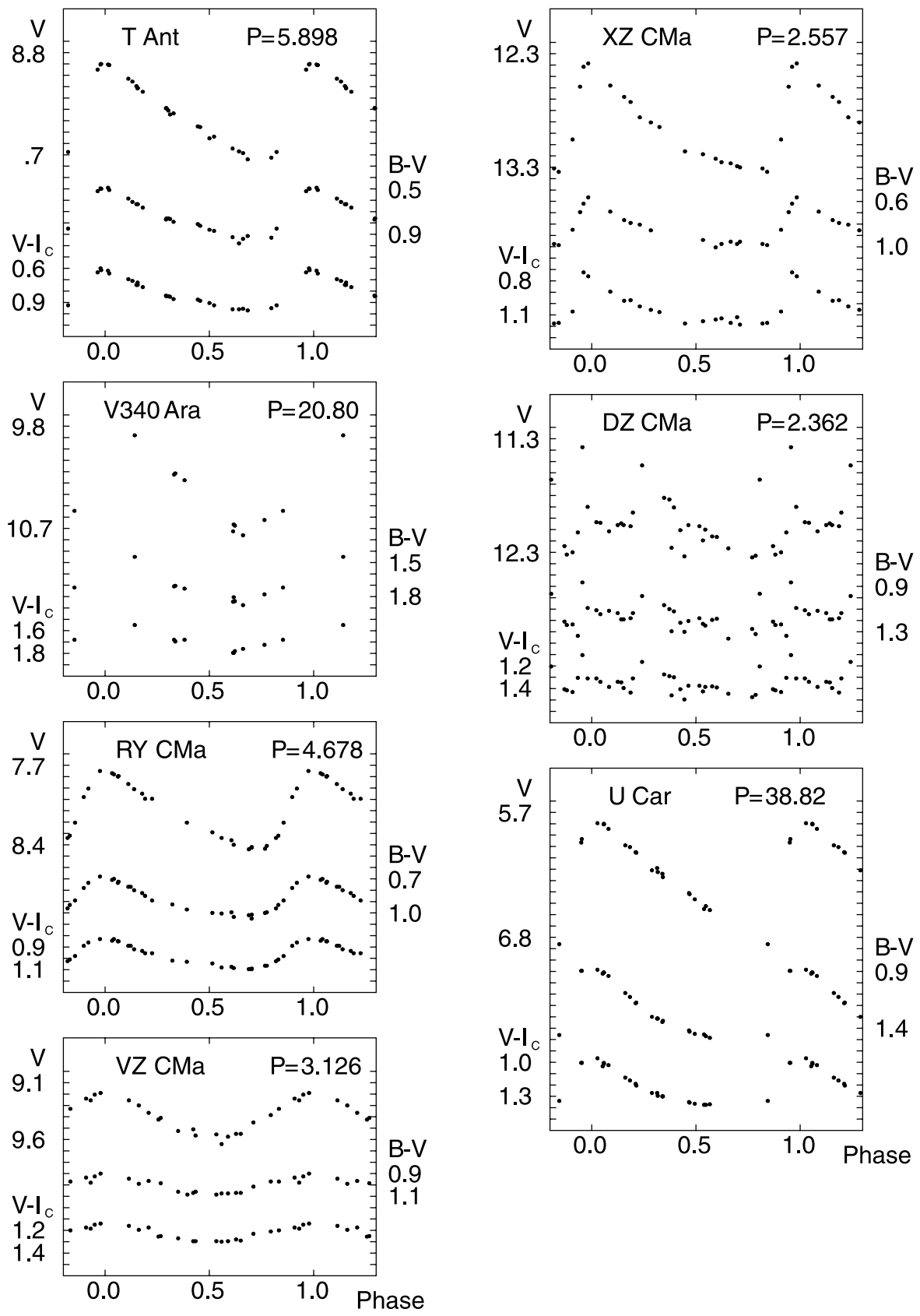

FIG. 1.- - Light and color curves for T Ant, V340 Ara, RY CMa, VZ CMa, XZ CMa, DZ CMa, and U Car 

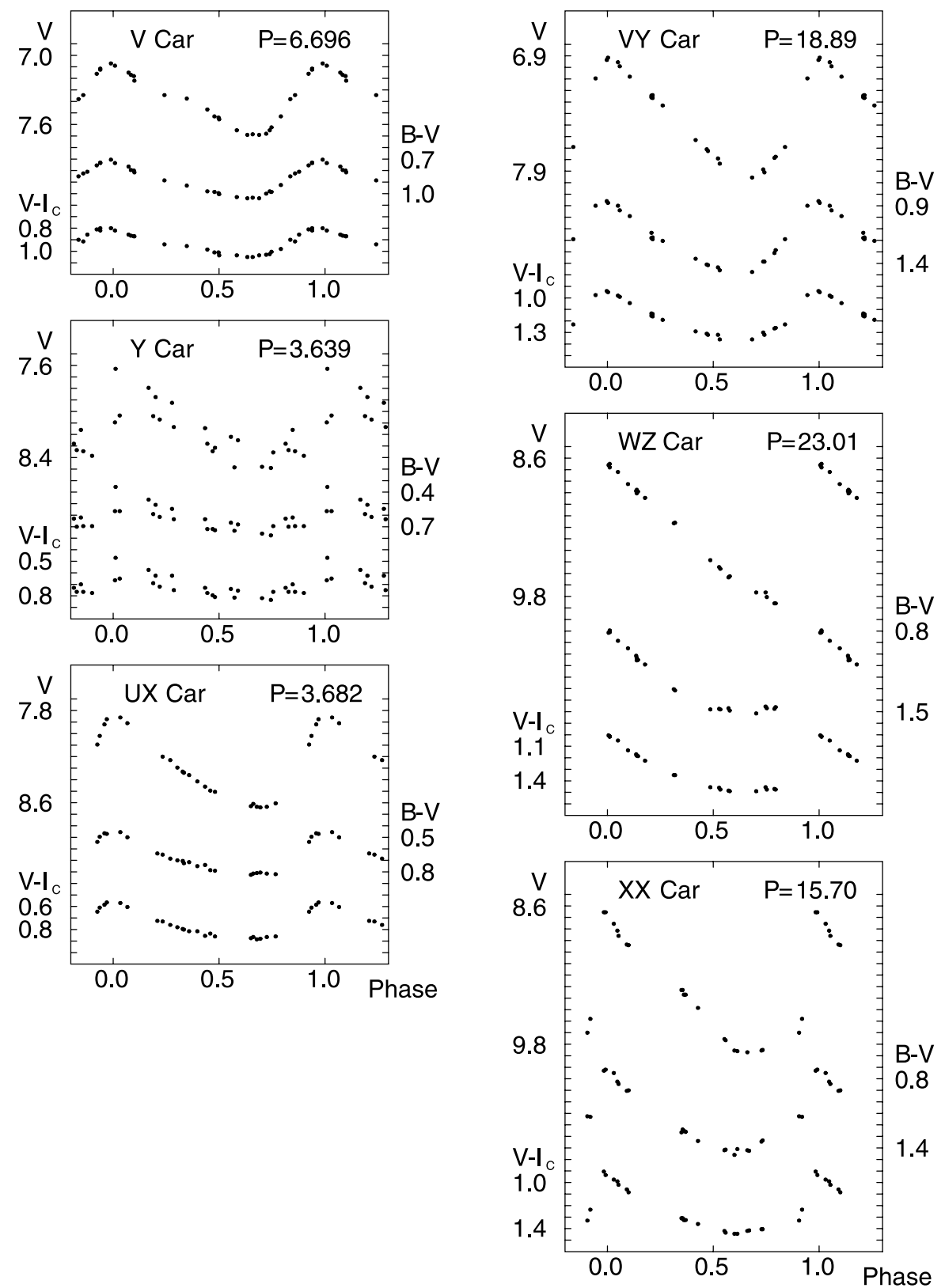

FIG. 2. - Light and color curves for V Car, Y Car, UX Car, VY Car, WZ Car, and XX Car 

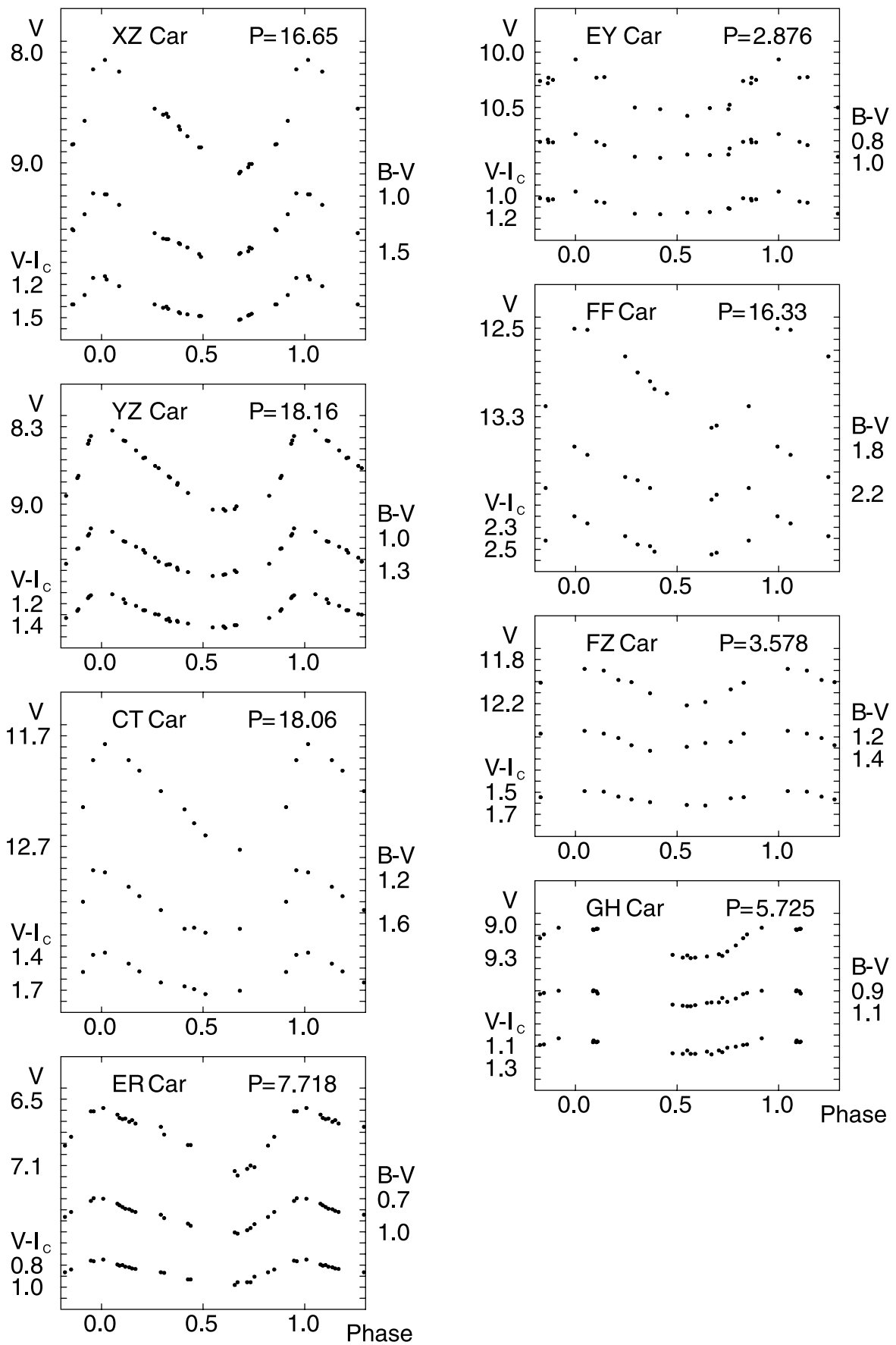

FIG. 3.- - Light and color curves for XZ Car, YZ Car, CT Car, ER Car, EY Car, FF Car, FZ Car, and GH Car 

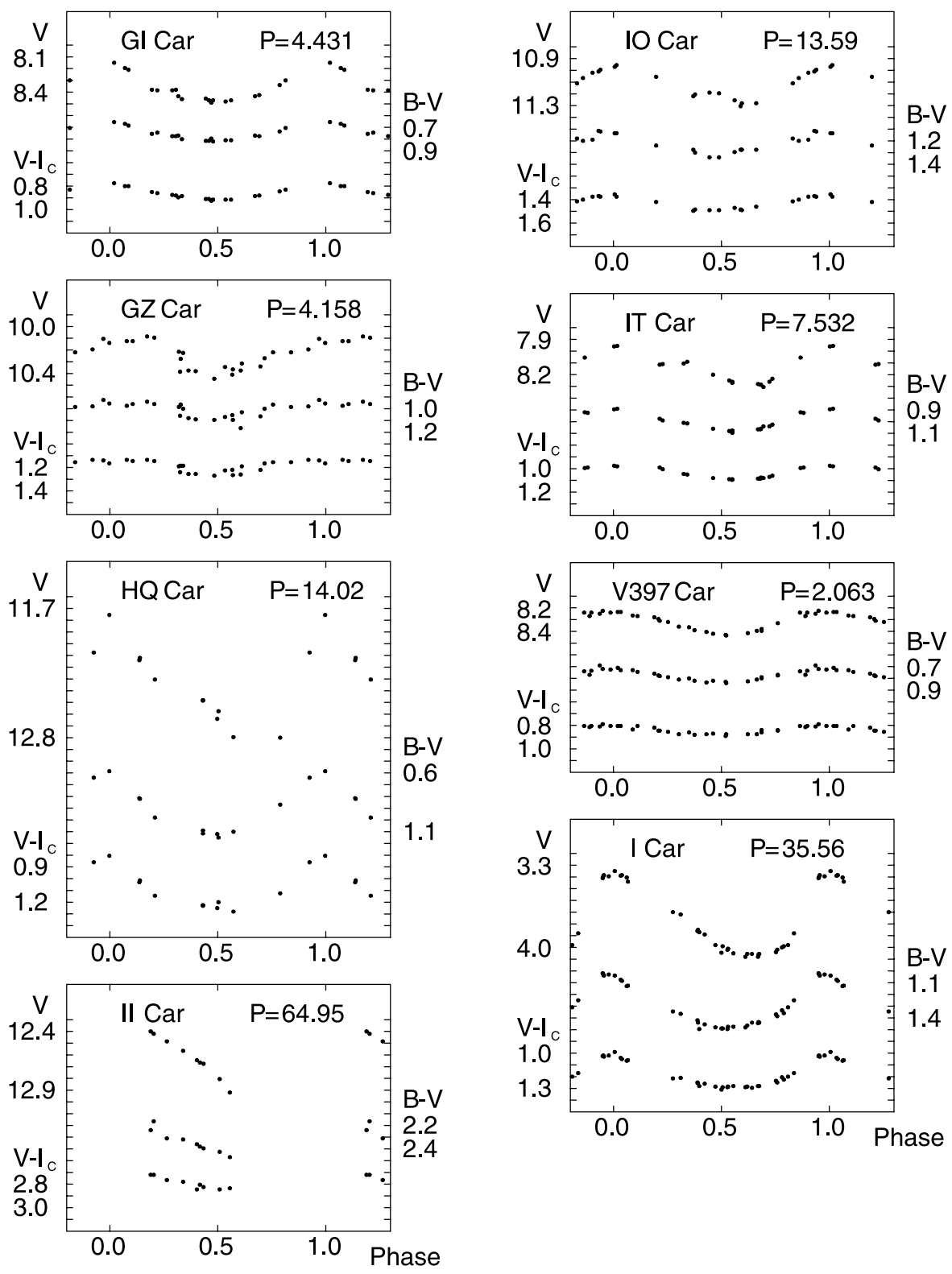

FIG. 4.- - Light and color curves for GI Car, GZ Car, HQ Car, II Car, IO Car, IT Car,V397 Car, and 1 Car 

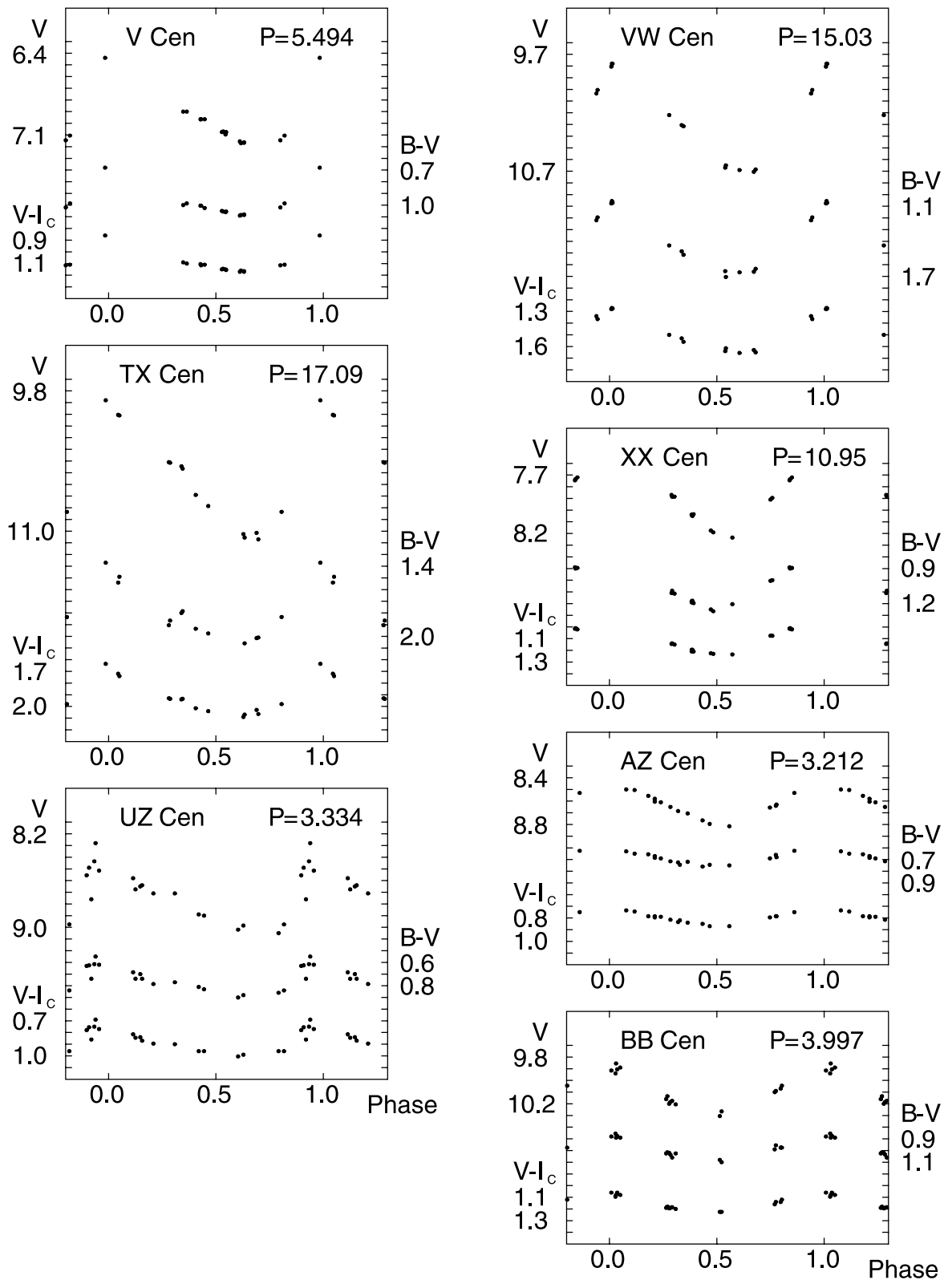

FIG. 5.- - Light and color curves for V Cen, TX Cen, UZ Cen, VW Cen, XX Cen, AZ Cen, and BB Cen 

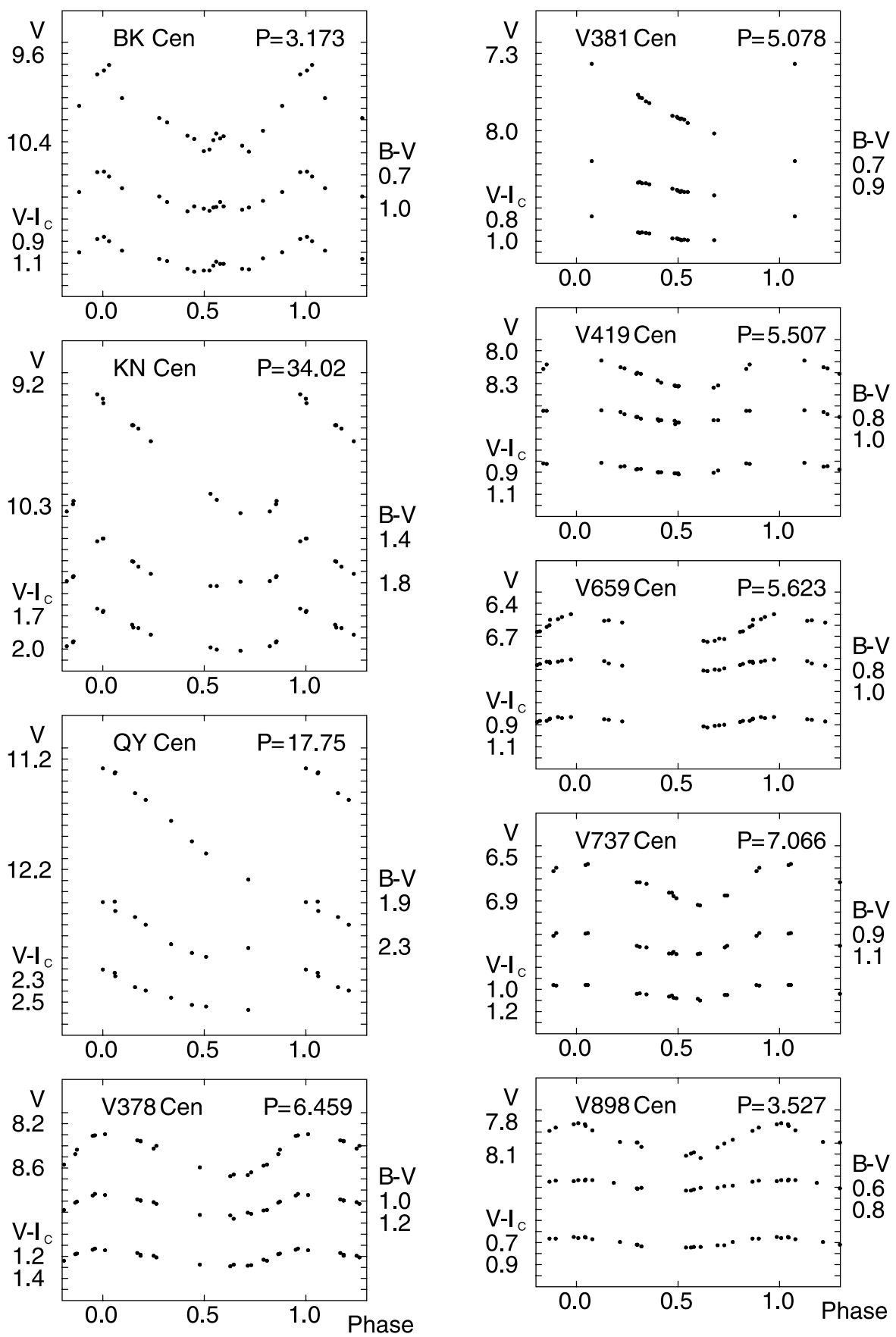

FIG. 6. - Light and color curves for BK Cen, KN Cen, QY Cen, V378 Cen, V381 Cen, V419 Cen, V659 Cen, V737 Cen, and V898 Cen 

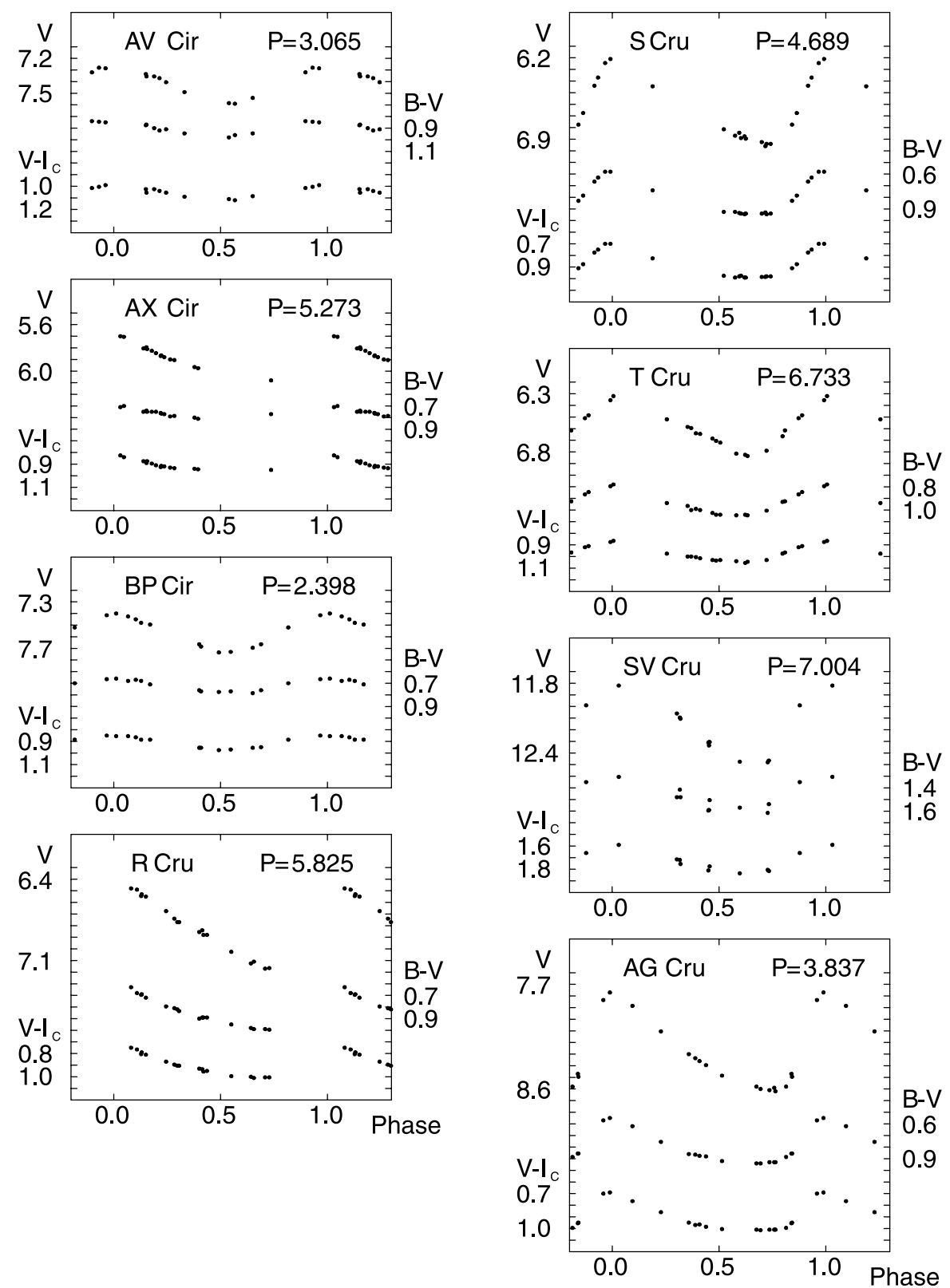

FIG. 7.- - Light and color curves for AV Cir, AX Cir, BP Cir, R Cru, S Cru, T Cru, SV Cru, and AG Cru 

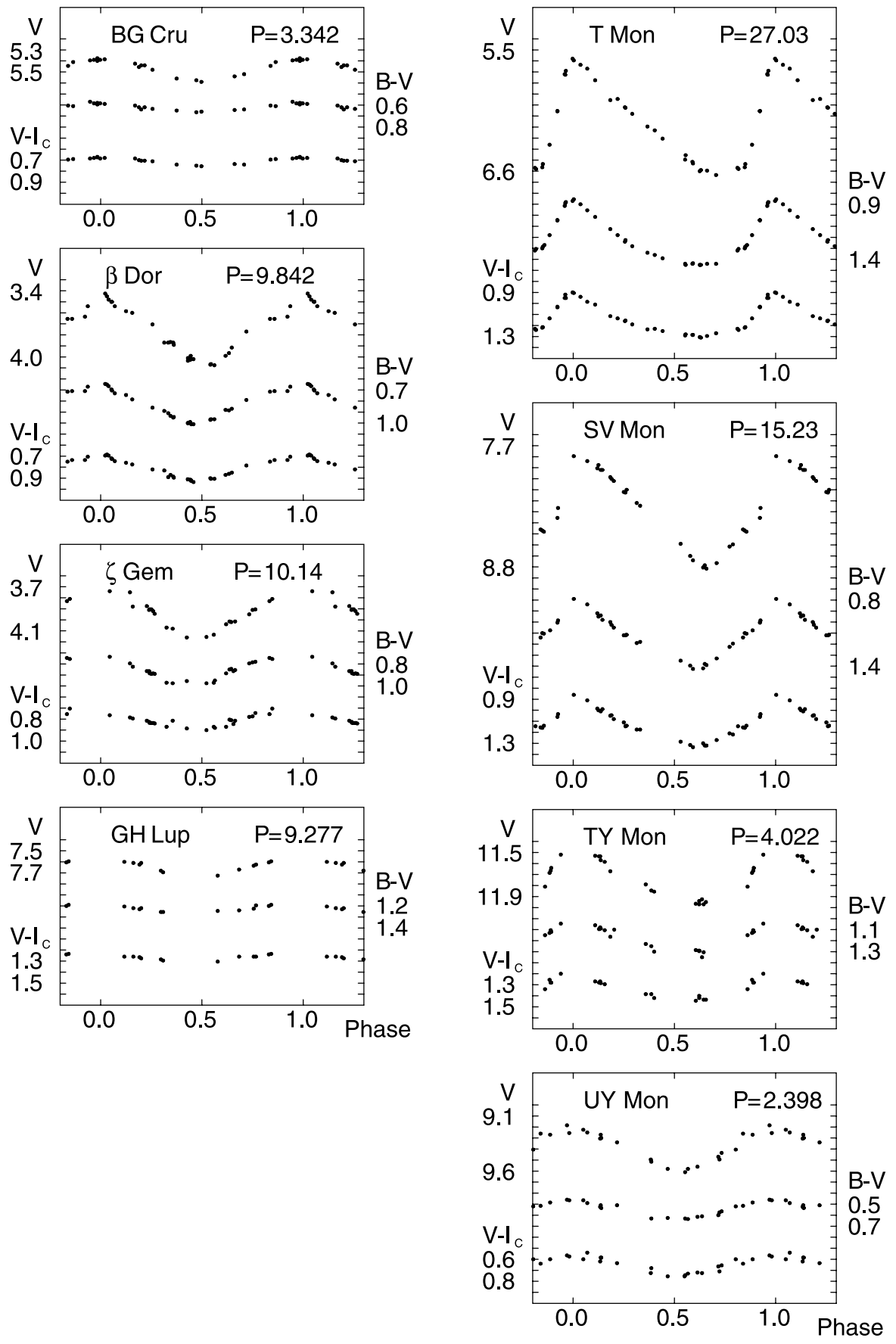

FIG. 8. - Light and color curves for BG Cru, $\beta$ Dor, $\zeta$ Gem, GH Lup, T Mon, SV Mon, TY Mon, and UY Mon 

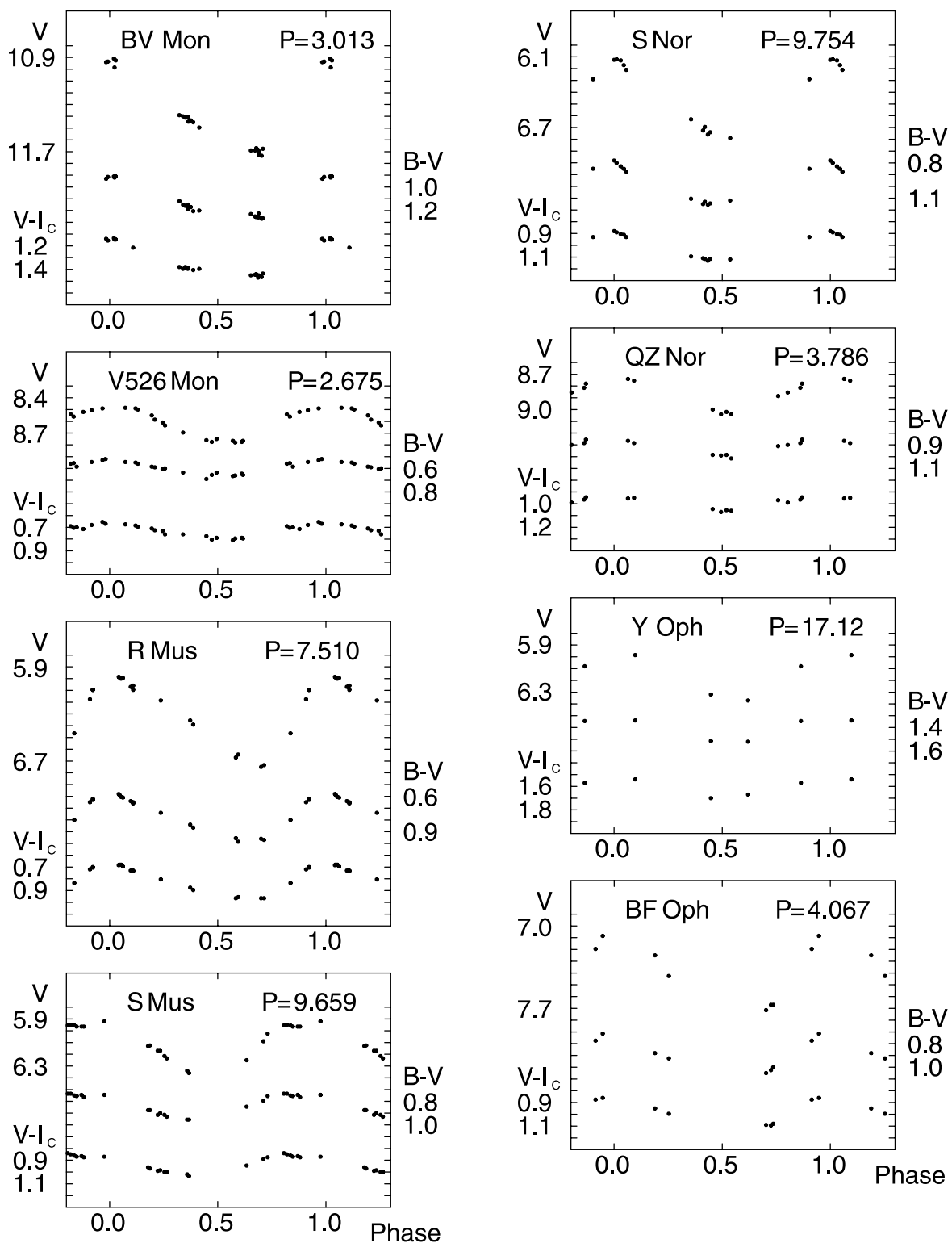

FIG. 9.- Light and color curves for BV Mon, V526 Mon, R Mus, S Mus, S Nor, QZ Nor, Y Oph, and BF Oph 

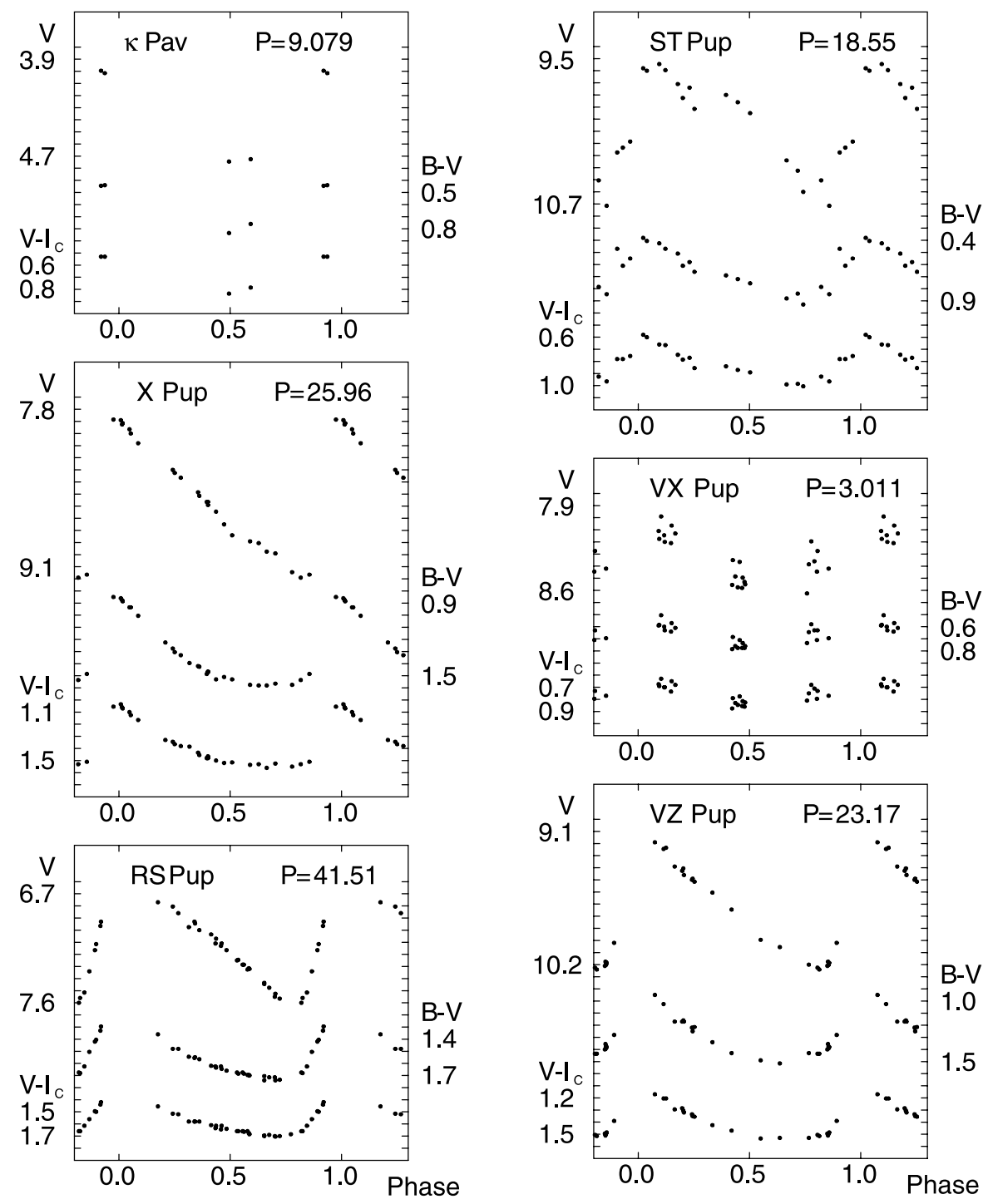

FIG. 10. - Light and color curves for $\kappa$ Pav, X Pup, RS Pup, ST Pup, VX Pup, and VZ Pup 

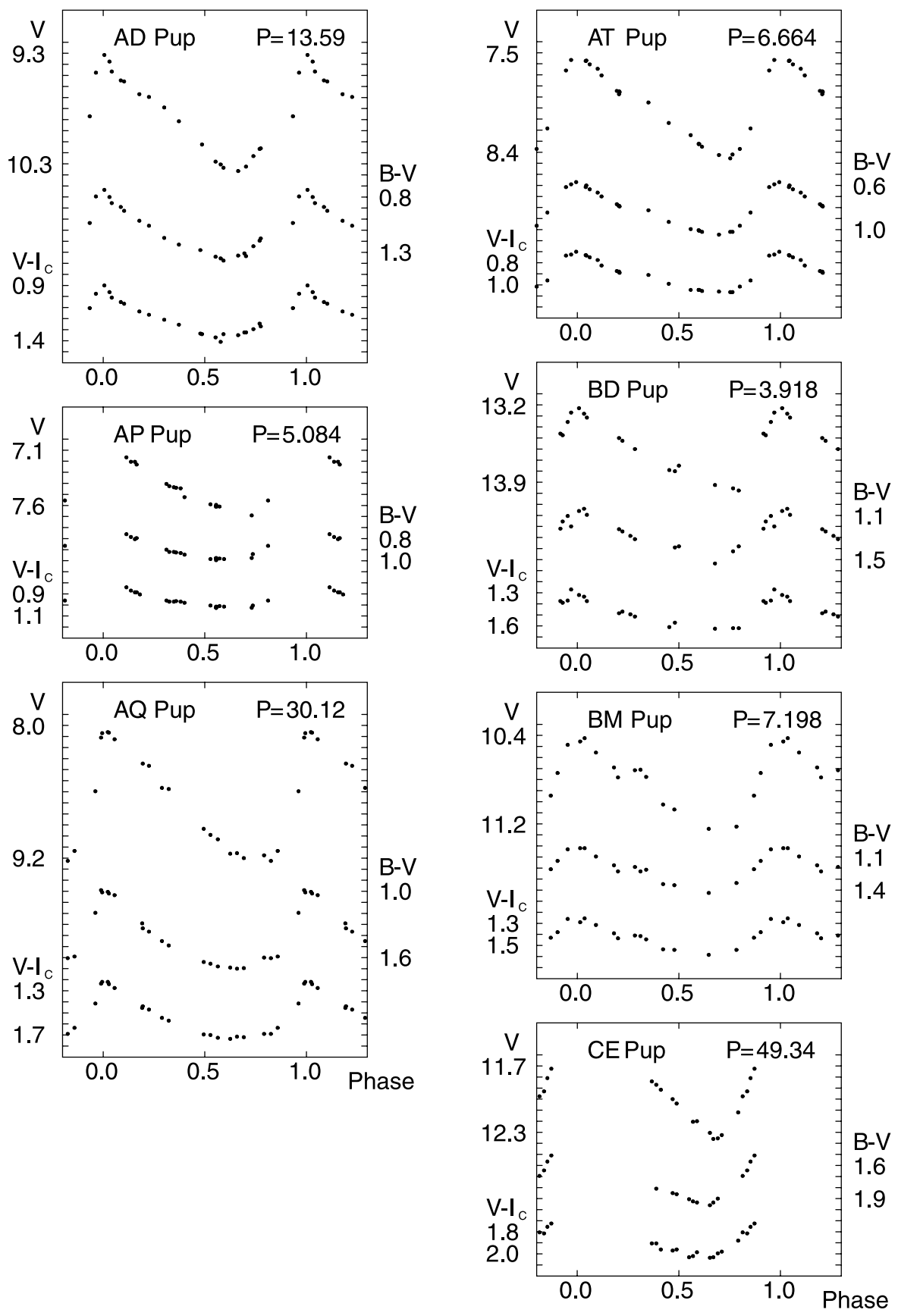

Fig. 11.-Light and color curves for AD Pup, AP Pup, AQ Pup, AT Pup, BD Pup, BM Pup, and CE Pup 

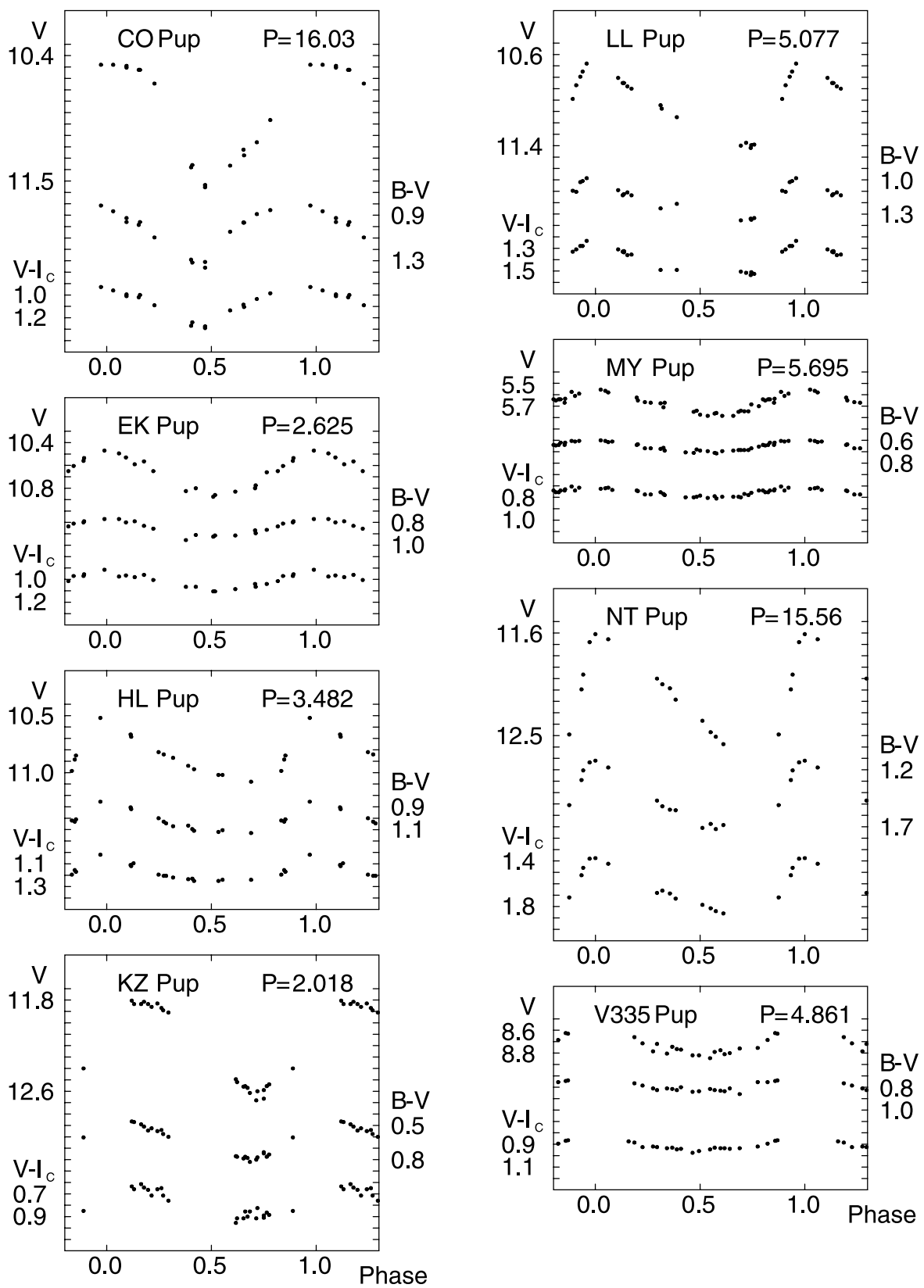

Fig. 12. - Light and color curves for CO Pup, EK Pup, HL Pup, KZ Pup, LL Pup, MY Pup, NT Pup, and V335 Pup 

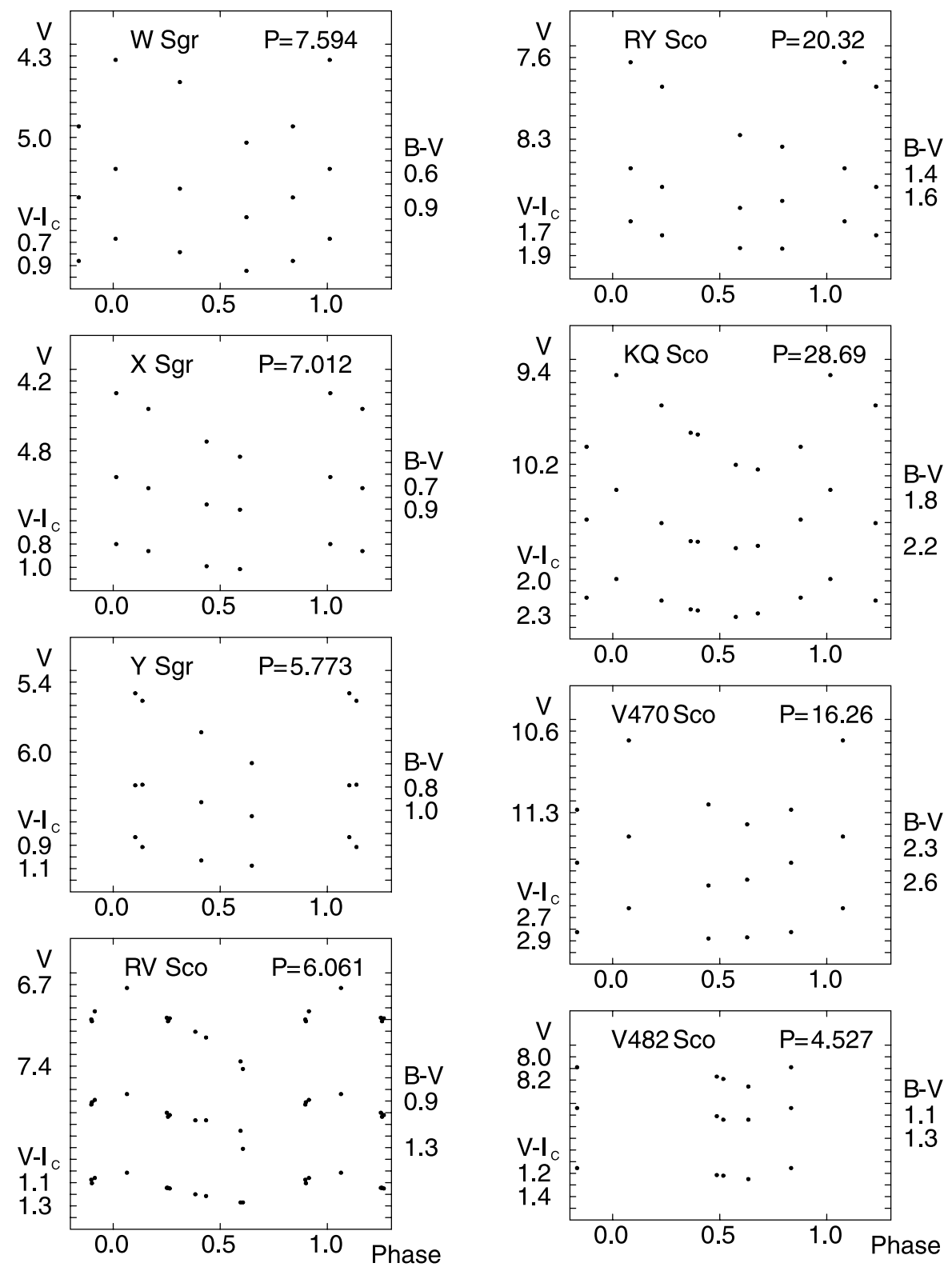

FIG. 13.- - Light and color curves for W Sgr, X Sgr, Y Sgr, RV Sco, RY Sco, KQ Sco, V470 Sco, and V482 Sco 

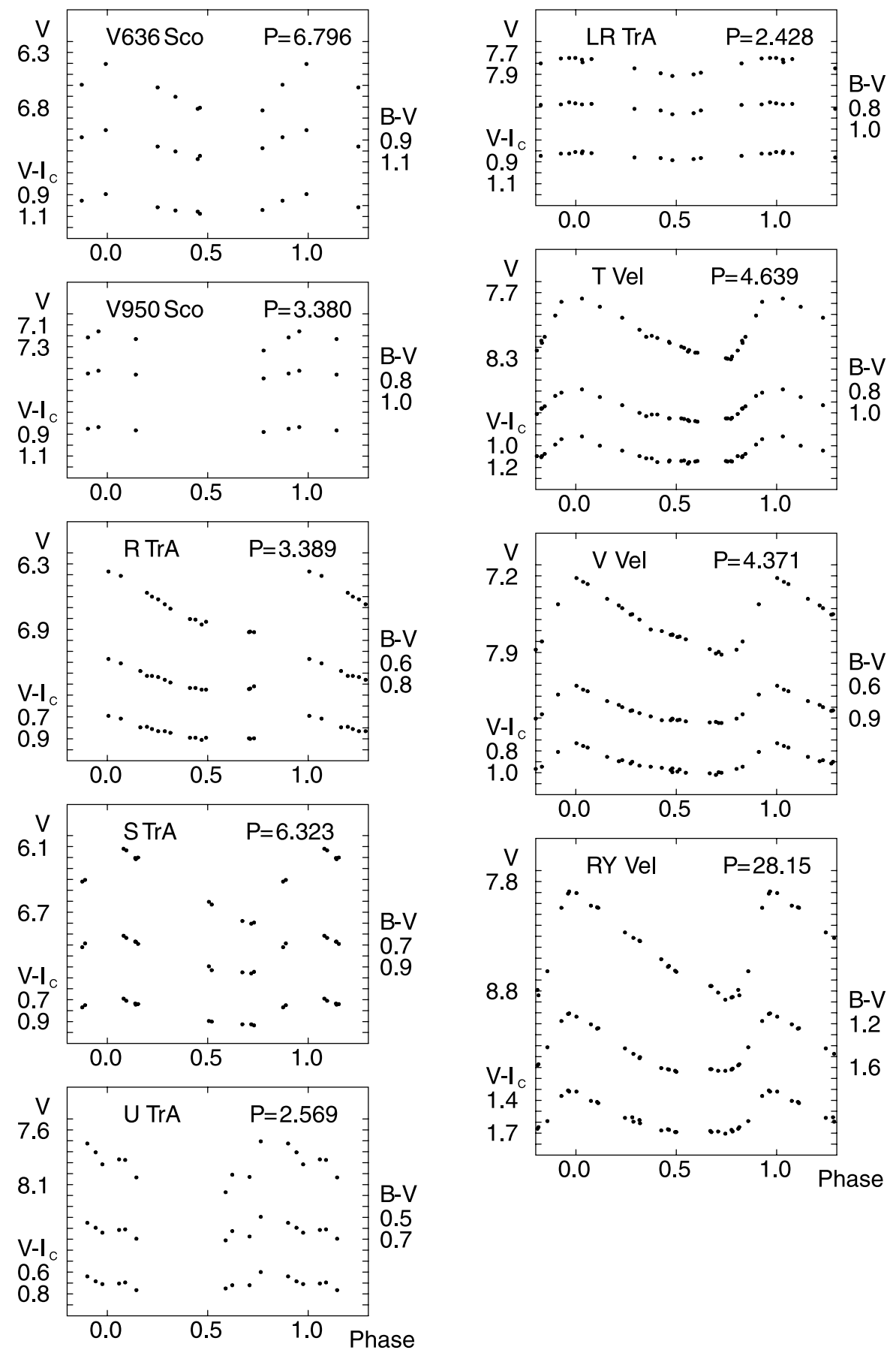

Fig. 14.- - Light and color curves for V636 Sco, V950 Sco, R TrA, S TrA, U TrA, LR TrA, T Vel, V Vel, and RY Vel 

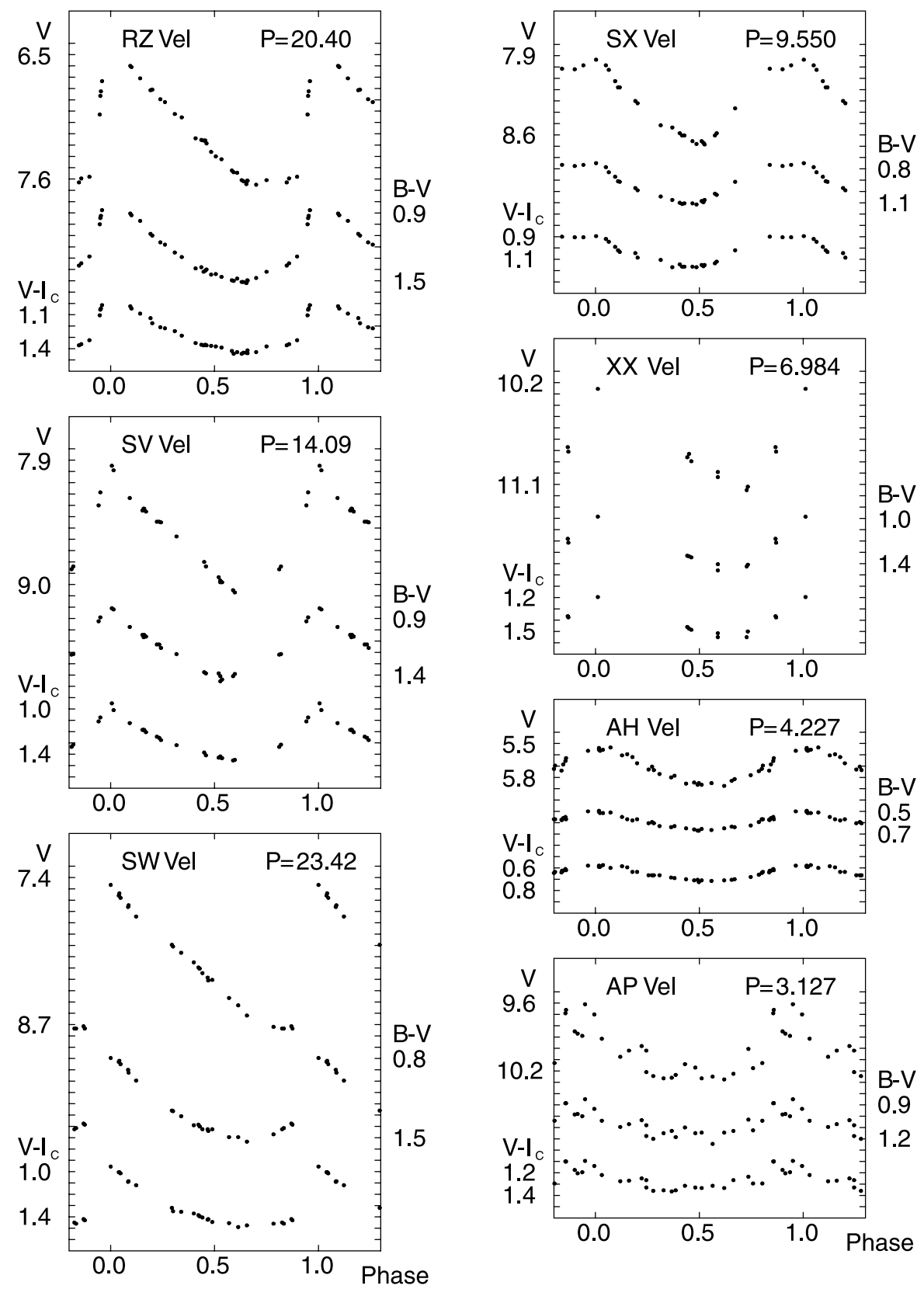

Fig. 15.--Light and color curves for RZ Vel, SV Vel, SW Vel, SX Vel, XX Vel, AH Vel, and AP Vel 

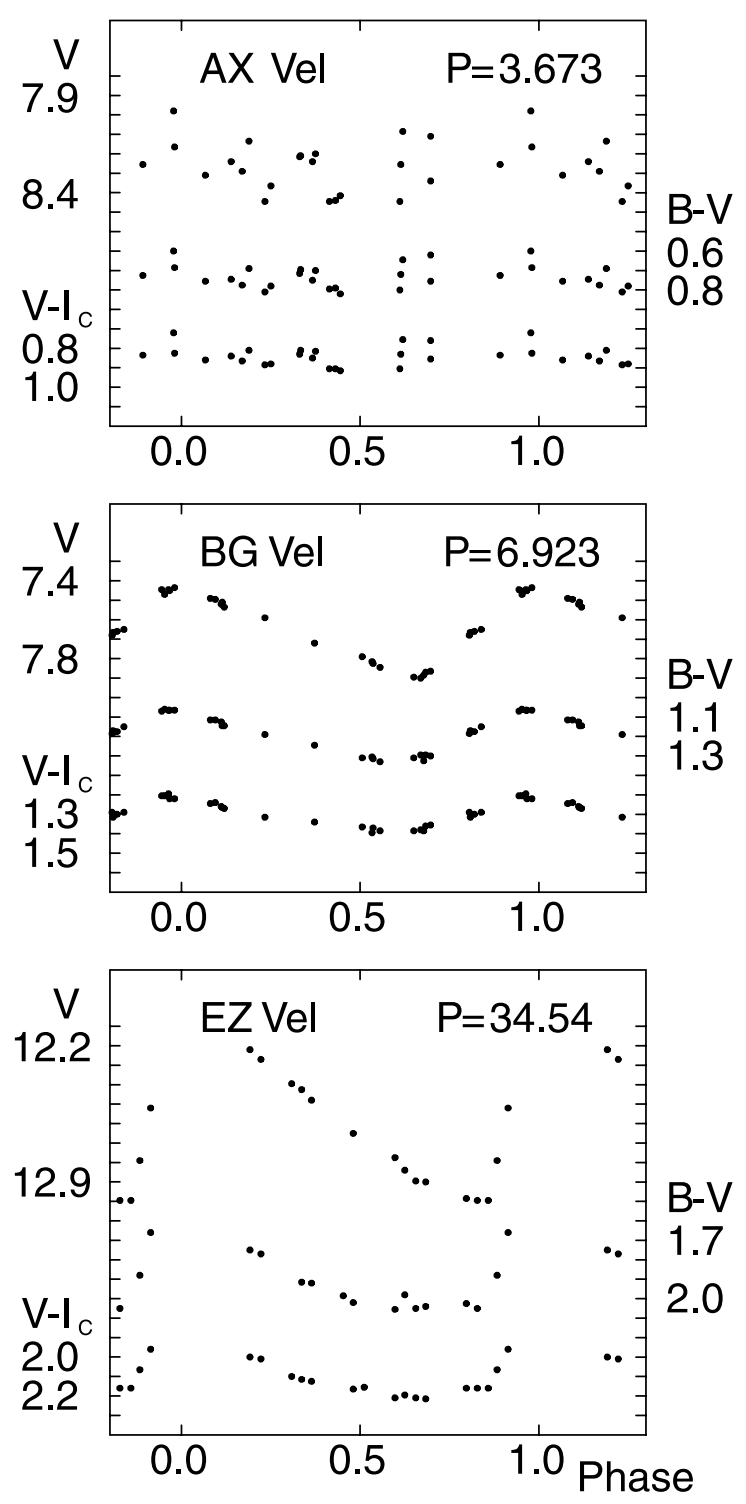

FIG. 16. - Light and color curves for AX Vel, BG Vel, and EZ Vel

Berdnikov, L. N. 1994, Astron. Lett., 20, 232

Berdnikov, L. N., \& Caldwell, J. A. R. 2001, in press

Berdnikov, L. N., \& Ignatova, V. V. 2000, ASP Conf. Ser. 203, The Impact of Large-Scale Surveys on Pulsating Star Research, ed. L. Szabados \& D. W. Kurtz (San Francisco: ASP), 244

Berdnikov, L. N., Ignatova, V. V., Caldwell, J. A. R., \& Koen, C. 2000, NewA, 4, 625

Berdnikov, L. N., Ignatova, V. V., Pastukhova, E. N., \& Turner, D. G. 1997a, Astron. Lett., 23, 177

Berdnikov, L. N., Ignatova, V. V., \& Vozyakova, O. V. 1997b, Astron. Astrophys. Trans., 14, 237

Berdnikov, L. N., \& Pastukhova, E. N. 1994a, Astron. Lett., 20, 479 1994b, Astron. Lett., 20, 720 1995, Astron. Lett., 21, 369

Berdnikov, L. N., \& Turner, D. G. 1995a, Astron. Lett., 21, 534 1995b, Astron. Lett., 21, 717

1998a, Astron. Astrophys. Trans., 16, 205

1998b, Astron. Astrophys. Trans., 16, 291

2000a, Astron. Astrophys. Trans., 18, 657

2000b, Astron. Astrophys. Trans., 18, 679

\section{REFERENCES}

Berdnikov, L. N., \& Vozyakova, O. V. 1995, Astron. Lett., 21, 308

Cousins, A. W. J. 1976, Mem. Soc. Astron. Italiana, 81, 25

Eddington, A. S., \& Plakidis, S. 1929, MNRAS, 90, 65

Menzies, J. W., Banfield, R. M., Cousins, A. J. W., \& Laing, J. D. 1989, South African Astron. Obs. Circ., 13, 1

Percy, J. R., Bezuhly, M., Milanowski, M., \& Zsoldos, E. 1997, PASP, 109, 264

Percy, J. R., \& Colivas, T. 1999, PASP, 111, 94

Percy, J. R., \& Hale, J. 1998, PASP, 110, 1428

Straizys, V. 1977, Multicolour Stellar Photometry (Vilnius: Mokslas)

Szabados, L. 1977, Mitt. Sternw. Ungarischen Akad. Wissenschaften 70 . 1980, Mitt. Sternw. Ungarischen Akad. Wissenschaften 76 . 1981, Mitt. Sternw. Ungarischen Akad. Wissenschaften 77 1989, Mitt. Sternw. Ungarischen Akad. Wissenschaften 94 1991, Mitt. Sternw. Ungarischen Akad. Wissenschaften 96

Turner, D. G. 1998, J. AAVSO, 26, 101

Turner, D. G., Billings, G. W., \& Berdnikov, L. N. 2001, PASP, 113, 715

Turner, D. G., Horsford, A. J., \& MacMillan, J. D. 1999, J. AAVSO, 27, 5 